\title{
The Performance of Trellis Coded $q$-ary PSK in Rayleigh Fading and Nakagami Fading with Dual Diversity Reception
}

\author{
C. Tellambura \\ Dept. of Digital Systems \\ Monash University \\ Wellington Road \\ Clayton, Victoria 3168 \\ Australia \\ Ph: +61399053296 \\ Fax: +61399053574 \\ Email: chintha@dgs.monash.edu.au
}

\begin{abstract}
An analytic technique is presented for computing the performance of trellis coded $q$-ary PSK in Rayleigh fading. A contour-integral expression for the pairwise error probability (PEP) is used to define a transfer function, enabling the union bound to be evaluated exactly for the ideal CSI (channel state information) case and imperfect-CSI case. The case of TCM in Nakagami fading and dual diversity reception is also analysed.
\end{abstract}

\section{INTRODUCTION}

The use of coding and diversity reception to enhance system performance in mobile communications systems has recently become feasible. Therefore, the performance of trellis coded modulation (TCM) schemes over mobile fading channels has recently received considerable attention. The average bit error probability $P_{b}$ is the most useful performance measure. Since explicit evaluation of $P_{b}$ appears impossible, the development of bounds on $P_{b}$ has been extensively researched [1-6]. The union bound technique is based on

$$
P_{b} \leq \frac{1}{k} \sum_{\mathbf{z}, \hat{\mathbf{z}} \in \mathcal{C}} a(\mathbf{z} \rightarrow \hat{\mathbf{z}}) P(\mathbf{z} \rightarrow \hat{\mathbf{z}})
$$

where $k$ is the number of input bits per encoding interval, $P(\mathbf{z} \rightarrow \hat{\mathbf{z}})$ is the PEP, $a(\mathbf{z} \rightarrow \hat{\mathbf{z}})$ is the number of associated bit errors, and $\mathcal{C}$ is the set of all legitimate code sequences. But the evaluation of even the union bound is difficult since $P(\mathbf{z} \rightarrow \hat{\mathbf{z}})$ requires residue calculations [3]. Thus, bounds on $P(\mathbf{z} \rightarrow \hat{\mathbf{z}})$ itself are used to compute (1), resulting in a weaker union bound. Recently, however, a method has been developed to compute the union bound exactly [6] for the ideal CSI case. This paper provides a more general method to evaluate the union bound exactly with ideal or imperfect CSI. Thus, the method is applicable to practical schemes such as differential detection and pilot tone aided detection. Performance evaluation for diversity reception of TCM in Nakagami fading is also provided.

\section{SYSTEM MODEL}

The received complex sample at time $n$ is

$$
y_{n}=\alpha_{n} z_{n}+v_{n}
$$

where $\alpha_{n}$ is the channel gain, and $v_{n}$ is an additive Gaussian noise sample. The following is used throughout the presentation:

$A 1 z_{n}$ is a $q$-ary Phase Shift Keying (PSK) symbol (i.e., $z_{n} \in\left\{e^{j 2 \pi k / q} \mid k=0,1, \ldots, q-1\right\}$ and $j=\sqrt{-1}$.

A2 Each $\alpha_{n}$ is a zero mean, complex, Gaussian random variable (RV). The $\alpha_{n}$ 's are independent (i.e., ideal interleaving/deinterleaving) and identically distributed RVs. A3 Each $\alpha_{n}$ remains constant during a symbol interval (i.e., non-selective slow fading)

A4 The receiver has some form of CSI given by $\hat{\alpha}_{n}$ which is a complex Gaussian RV. The correlation coefficient between $\alpha$ and $\hat{\alpha}_{n}$ is $\mu$.

If $\mu=1$, ideal CSI exists. For practical channel estimators $|\mu| \leq 1$. The more $\mu$ deviates from unity, the larger is the performance penalty.

\section{Patrwise Error Event Probability}

Consider two codewords $\mathbf{z}=\left\{z_{1}, z_{2}, \ldots, z_{N}\right\}$ and $\hat{\mathbf{z}}=\left\{\hat{z}_{1}, \hat{z}_{2}, \ldots, \hat{z}_{N}\right\}$ of length $N$. The Viterbi decoder computes the path metrics $[3,(9)]$ and selects $\mathbf{z}$ over $\hat{\mathbf{z}}$ according to the path metric difference $D$. The characteristic function of $D, \phi(j \omega)=E\left[e^{j \omega D}\right]$, can be written as $[3,(23)]$

$$
\phi(j \omega)=\prod_{n \in \eta} \frac{\Delta_{n}}{\omega^{2}-j \omega+\Delta_{n}}
$$

where $\Delta_{n} \triangleq\left[1+\left(1-|\mu|^{2}\right) \gamma_{s}\right] /\left(|\mu|^{2}\left|z_{n}-\hat{z}_{n}\right|^{2} \gamma_{s}\right), \eta \triangleq$ $\left\{n \mid z_{n} \neq \hat{z}_{n}, n=1, \ldots, N\right\}$, and $\gamma_{s}=\bar{E}_{s} / N_{0}$ is the average signal-to-noise ratio. It can be shown that $[7$, $(\mathrm{B}-4)]$

$$
P(\mathbf{z} \rightarrow \hat{\mathbf{z}})=\frac{-1}{2 \pi j} \int_{-\infty+j \epsilon}^{\infty+j \epsilon} \frac{\phi(j \omega)}{\omega} d \omega
$$

where $\epsilon$ is a small positive number. An explicit expression for $P(\mathbf{z} \rightarrow \hat{\mathbf{z}})$, which cannot be used with the transfer function approach, can be obtained by solving for the residues of the contour integral [3]. However, a transfer function can be defined with the factors of $\phi(j \omega)$. 


\section{UNION BOUND}

Consider the evaluation of the union bound (1). Let $\mathbf{Z}=\left(Z_{1}, Z_{2}, \ldots\right)$ be a vector of formal variables. Define the generating function of the form

$$
T(\mathbf{Z}, I)=\sum_{\mathbf{z}, \hat{\mathbf{z}} \in \mathcal{C}} I^{a(\mathbf{z} \rightarrow \overline{\mathbf{z}})} \prod_{n \in \eta} Z_{n}
$$

where $I$ is another formal variable. Moreover, let

$$
D_{n}(\omega) \triangleq \frac{\Delta_{n}}{\omega^{2}-j \omega+\Delta_{n}} .
$$

The number of distinct values that $D_{n}(\omega)$ can take depends on the size of the signal constellation. The transfer function $T(\mathbf{D}(\omega), I)$ can be determined by a signal flow graph with the branch labels $I^{v} D_{n}(\omega)$ for uniform trellis codes [8]. By contrast, for the union-Chernoff bound, the branches are labeled with $I^{v}\left(1+1 /\left(4 \Delta_{n}\right)\right)^{-1}$.

Combining (1), (3) and (4), and using the standard analysis [9], it follows that

$$
P_{b} \leq\left.\frac{-1}{j 2 \pi k} \frac{\partial}{\partial I}\left\{\int_{-\infty+j \epsilon}^{\infty+j \epsilon} \frac{T(\mathbf{D}(\omega), I)}{\omega} d \omega\right\}\right|_{I=1}
$$

where the partial derivative can be computed with [9, 4.5.9]. While this integral has no analytical solution in general, its numerical computation poses little difficulty. Since $|T(\mathbf{D}(\omega), I)| \rightarrow 0$ as $|\omega| \rightarrow \infty$, simple techniques such as the Simpson method are adequate.

Example 1: Consider the reception of rate 1/2, twostate trellis-coded QPSK in Rayleigh fading. This coded modulation is analyzed in [10, Example 9.1] for ideal CSI. Using branch label gains, $D_{n}(\omega)$, the transfer function becomes

$$
T(\mathbf{D}(\omega), I)=\frac{I \Delta_{2} \Delta_{4}}{\left(\omega^{2}-j \omega+\Delta_{4}\right)\left(\omega^{2}-j \omega+(1-I) \Delta_{2}\right)}
$$

where $\Delta_{2}$ and $\Delta_{4}$ are obtained with $\left|z_{n}-\hat{z}_{n}\right|^{2}$ equal to 2 and 4 , respectively. Substituting this in (6), carrying out the integration, and evaluating the derivative at $I=1$, one has

$$
\begin{aligned}
P_{b} \leq & 1-\frac{\left(1+\left(1-|\mu|^{2}\right) \gamma_{s}\right)}{2|\mu|^{2} \gamma_{s}}+\frac{3\left(1+\left(1-|\mu|^{2}\right) \gamma_{s}\right)^{2}}{4|\mu|^{4} \gamma_{s}^{2}} \\
& -|\mu| \sqrt{\frac{\gamma_{s}}{1+\gamma_{s}}} .
\end{aligned}
$$

This is the union bound for this coded modulation. If differential detection is used in a flat fading land mobile channel, the correlation coefficient $\mu$ is given in $[3,(32)]$ as a function of the normalized maximum Doppler frequency $f_{d} T$. Fig. 1 shows (7) for several $f_{d} T$ values. Unless $f_{d} T=0$, error floors exist.

Example 2: Consider the trellis coded 8PSK scheme given in [2, Fig. 5]. Its transfer function [2, (19)] can be defined with the weight profiles obtained using $D_{n}(\omega)$. Consider pilot tone aided detection with $\mu$ given in [3, (40)]. Assume that the bandwidth of the pilot tone filter be $2 f_{d}$ and the power-split ratio be $\sqrt{2 f_{d} T}[5,(17)]$. Fig. 2 shows the simulation results and the union bound (6). At $P_{b} \approx 10^{-4}$, the simulation results are within $0.2,1.0$, and $1.5 \mathrm{~dB}$ of the union bound for $f_{d} T$ of $0,0.02$, and 0.04 , respectively.

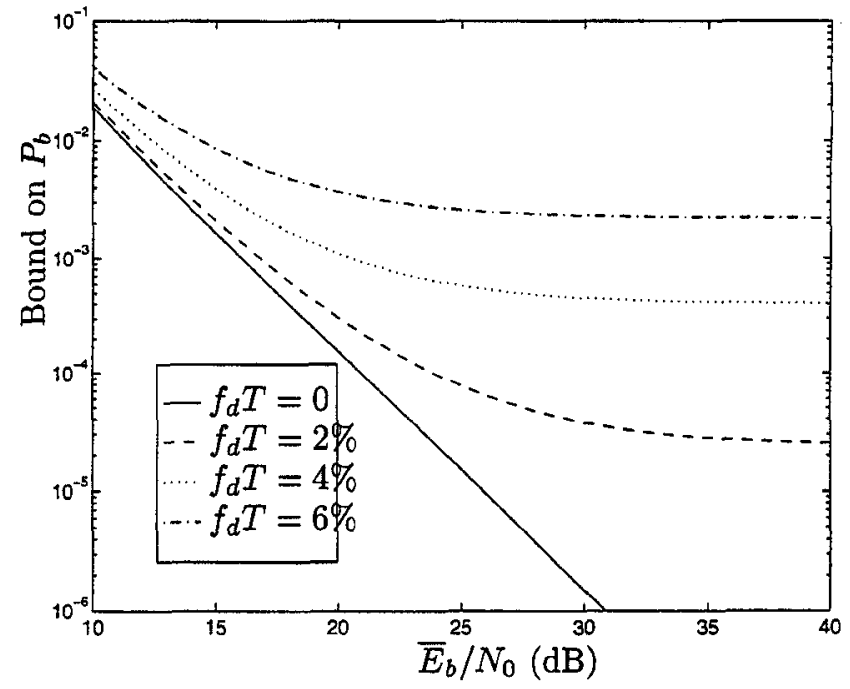

Fig. 1. Bit error performance of rate $1 / 2$ trellis-coded QPSK (differential detection) for fast Rayleigh fading.

\section{DUAL DIVERSITY MRC RECEPTION IN NAKAGAMI FADING}

For brevity, some results in [11] are taken as the starting point of the development here. From a theoretical point of view, the Nakagami distribution is more general than the Rayleigh model and may be used to model a wide variety of fading scenarios.

Assuming maximal ratio combining (MRC) and independent fading branches, the PEP is given as [11, eq. (7)]

$$
P(\mathbf{z} \rightarrow \hat{\mathbf{z}})=\frac{1}{\pi} \int_{0}^{\pi / 2} \prod_{n \in \eta} \prod_{k=1}^{2}\left(1+\frac{\gamma_{k}\left|z_{n}-\hat{z}_{n}\right|^{2}}{4 \sin ^{2} \phi}\right)^{-m_{k}} d \phi
$$

where $m_{k}$ is the Nakagami fading index and $\gamma_{k}$ is the average signal-to-noise ratio for the $k$ th diversity branch. Similar to Section 4, let

$$
D_{n}(\phi) \triangleq \prod_{k=1}^{2}\left(1+\frac{\gamma_{k}\left|z_{n}-\hat{z}_{n}\right|^{2}}{4 \sin ^{2} \phi}\right)^{-m_{k}}
$$

The number of distinct values that $D_{n}(\phi)$ can take depends on the size of the signal constellation. As before, the transfer function $T(\mathbf{D}(\phi), I)$ can be determined by a signal flow graph with the branch labels $I^{v} D_{n}(\phi)$ for uniform trellis codes [8].

Combining (1), (8) and (4), and using the standard analysis [9], it follows that

$$
P_{b} \leq\left.\frac{1}{k \pi} \frac{\partial}{\partial I}\left\{\int_{0}^{\pi / 2} T(\mathbf{D}(\phi), I) d \phi\right\}\right|_{I=1}
$$

where the partial derivative can be computed with $[9$, 4.5.9]. While this integral has no analytical solution in general, its numerical computation poses little difficulty, and simple techniques such as the Simpson method are adequate.

Example 3: Consider the reception of rate 1/2, twostate trellis-coded QPSK in Nakagami fading with dual 
MRC reception. This coded modulation is analyzed in [10, Example 9.1] for ideal CSI. In the absence of fading, the transfer function is given as

$$
T(D, I)=\frac{4 a c}{1-2 b} \quad a=\frac{I}{2} D^{4} \quad b=\frac{I}{2} D^{2} \quad c=\frac{1}{2} D^{2} .
$$

In this case, using branch label gains, $D_{n}(\phi)$, with

$$
\begin{aligned}
a & =\frac{I}{2} \prod_{k=1}^{2}\left(1+\frac{\gamma_{k}}{\sin ^{2} \phi}\right)^{-m_{k}} \\
b & =\frac{I}{2} \prod_{k=1}^{2}\left(1+\frac{\gamma_{k}}{2 \sin ^{2} \phi}\right)^{-m_{k}} \\
c & =\frac{1}{2} \prod_{k=1}^{2}\left(1+\frac{\gamma_{k}}{2 \sin ^{2} \phi}\right)^{-m_{k}}
\end{aligned}
$$

the transfer function becomes

$$
T(\mathbf{D}(\phi), I)=\frac{I \prod_{k=1}^{2}\left(1+\frac{\gamma_{k}}{\sin ^{2} \phi}\right)^{-m_{k}}\left(1+\frac{\gamma_{k}}{2 \sin ^{2} \phi}\right)^{-m_{k}}}{1-I \prod_{k=1}^{2}\left(1+\frac{\gamma_{k}}{2 \sin ^{2} \phi}\right)^{-m_{k}}}
$$

where $\Delta_{2}$ and $\Delta_{4}$ are obtained with $\left|z_{n}-\hat{z}_{n}\right|^{2}$ equal to 2 and 4, respectively. Substituting this in (10), carrying out the integration, and evaluating the derivative at $I=$ 1 , one has the union bound for this coded modulation. Fig. 3 shows some performance curves for several values of Nakagami fading index $(m=0.5,1,2)$. It is seen that increasing values of $m_{k}$ lead to improved performance.

\section{CONCLUSIONS}

A novel method to compute the union bound (exactly) on the average bit error probability of TCM schemes over Rayleigh fading channels has been derived. The contour integral can be evaluated with simple numerical algorithms. The method facilitates the evaluation of the system performance in fading channels where ideal coherent detection is unrealizable. The basic results have also been extended to the case of dual diversity reception and Nakagami fading.

\section{REFERENCES}

[1] D. Divsalar and M. K. Simon, "Trellis-coded modulation for 4800 to 9600 bps transmission over a fading satellite channel," IEEE J. Select. Areas. Commun., vol. 5, pp. 162-175, Feb. 1987.

[2] R. G. McKay, E. Biglieri, and P. J. McLane, "Error bounds for Trellis-Coded MPSK on a fading mobile satellite channel," IEEE Trans. Commun., vol. 39, pp. 1750-1761, Dec. 1991.

[3] J. K. Cavers and P. Ho, "Analysis of the error performance of trellis coded modulations in Rayleigh fading channels," IEEE Trans. Commun., vol. 40, pp. 74-83, Jan. 1992.

[4] S. B. Slimane and T. Le-Ngoc, "Tight bounds on the error probability of coded modulation schemes in Rayleigh fading channels," IEEE Trans. Veh. Technol., vol. 44, pp. 121-129, Jan. 1995.

[5] K. Chan and A. Bateman, "The performance of referencebased $M$-ary PSK with trellis coded modulation in Rayleigh fading," IEEE Trans. Veh. Technol., vol. 41, pp. 190-198, May 1992.

[6] C. Tellambura, "Evaluation of the exact union bound for trellis coded modulations over fading channels," IEEE Trans. Commun., Dec. 1996.

[7] J. G. Proakis, Digital Communications. New York: McGraw Hill, 3rd ed., 1995.

[8] E. Zehavi and J.K. Wolf, "On the performance of trellis codes," IEEE Trans. Inform. Theory., vol. 33, pp. 196-202, Mar. 1987.

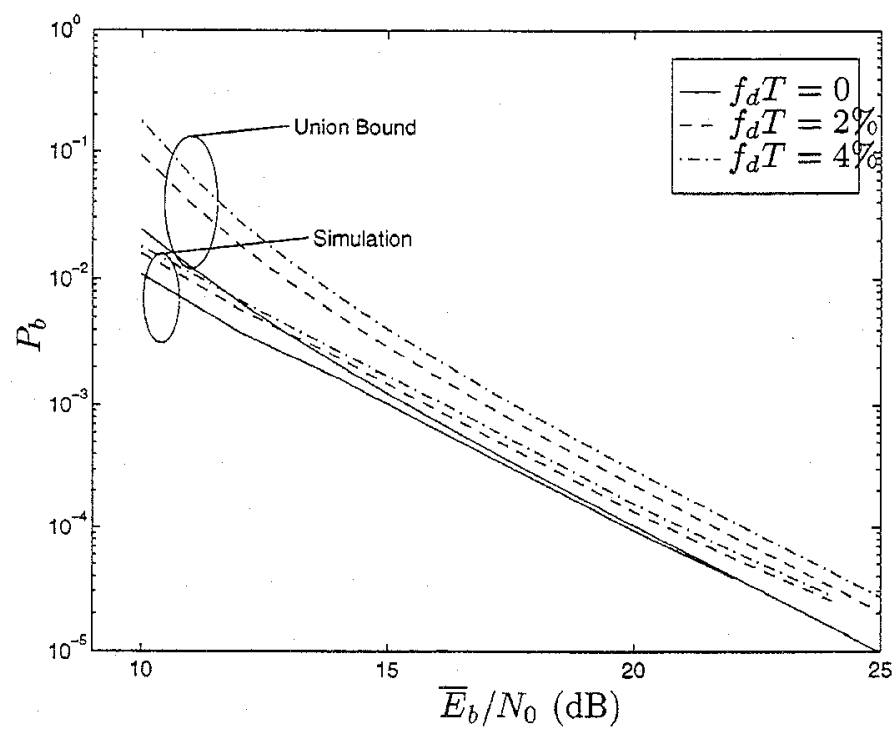

Fig. 2. Bit error performance of rate $2 / 3$, four-state, trellis-coded 8PSK for fast Rayleigh fading and pilot tone aided detection.

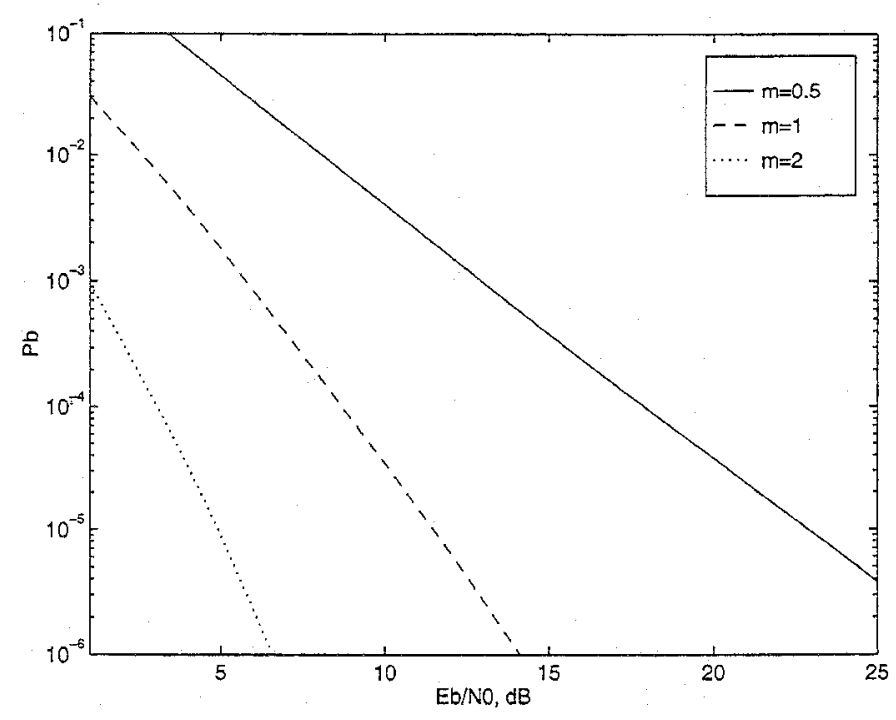

Fig. 3. Bit error performance of rate $1 / 2$ treliis-coded QPSK in dual MRC reception; independent and identical fading branches with Nakagami fading.

[9] A. J. Viterbi and J. K. Omura, Principles of Digital Communication and Coding. New York: McGraw-Hill, 1979.

[10] E. Biglieri, P. J. McLane, M. K. Simon and D. Divsalar, Introduction to Trellis-Coded Modulation with Applications. New York: McMillan, 1991.

[11] G. Femenias and I. Furio, "Dual MRC reception of TCMMPSK signals over Nakagami fading channels," Electron. Lett., vol. 32, pp. 1752-1753, Sept. 1996. 